\title{
The obesity impact on fertility
}

\begin{abstract}
Introduction: The rising prevalence of child obesity has a profound impact on worldwide health, nowadays, the children that have an increased body mass index (BMI) associated with: diet, sedentarism and genetic influence, results in obese child since early ages. The problem has been demonstrated, that since embryo stage, uterus environment promotes expression of genes that predispose degenerative diseases such as diabetes, hypertension, and obesity. Obesity during the childhood continue to puberty create a proinflammatory microenvironment that impact germ cells directly. Male obesity is associated with compromised spermatogenesis and spermiogenesis due to hormone levels alterations. Female obesity is associated to ovulatory disorders, poorer outcomes in fertility treatment and requires higher doses of medications for ovulation induction. Pregnancy rate vary among studies however there is a clear association between obesity and early pregnancy loss. There are several mechanisms why obesity causes fertility problems. The increase of leptin concentration, decrease of adiponectin levels, variation of kiss pectin expression to reach puberty all affect hormonal level and influence germ cell development.
\end{abstract}

Objectives: To describe the impact of obesity in childhood and the possible negative prognosis on future fertility.

Methods: Literature search was performed in PubMed from January 2000 to March 2017 using the search terms child obesity and: infertility, spermiogenesis, spermatogenesis, ovulation, oocyte, and pregnancy rates.

Conclusion: Obesity affects fertility since intrauterine development all the way though adult life. Obesity has multiple effects that involve fertility that creates a constant proinflammatory microenvironment in germ cell that have a negative impact on reproduction in adults of both genders.

Keywords: child obesity, fertility prognosis, germ cells
Volume 5 Issue 3 - 2018

Jose Manuel Lozano Sanchez, ${ }^{2}$ Hector Godoy Morales, ${ }^{2}$ Paola Berenice Merchand Alvarez, ${ }^{2}$ Jose Alfonso Gutierrez Frusch, ${ }^{2}$ Ricardo Mera Mejia, ${ }^{2}$ Luis Felipe Montano Estrada, ${ }^{3}$ Erika Patricia Rendon Huerta, ${ }^{3}$

\section{Zoraida Axtle Serrano'}

'CSIHM Comprehensive Health Center for Men and Women, Mexico

${ }^{2}$ ART Reproduction, Mexico

${ }^{3}$ Faculty of Medicine, National Autonomous University of Mexico, Mexico

Correspondence: Zoraida Axtle Serrano, Department of Nutrition and Metabolism, CSIHM Comprehensive Health Center for Men and Women, Tehuantepec 25 I cons 506, México City, México, Tel (0I I)554I652834, Email draaxtle@csihm.com

Received: April 19, 2017| Published: June 21, 2018

\section{Introduction}

Obesity is defined as abnormal or excessive fat accumulations that may impair health include fertility. The most common way to evaluate fat accumulation is through the Body mass index (BMI) which is calculated using person's weight in kilograms divided by the square of his height in meters $\left(\mathrm{kg} / \mathrm{m}^{2}\right)$.

For ages, we classified in:

i. Adults: WHO defines obesity as a BMI greater than or equal to 30 .

ii. Children under 5 years of age: Obesity is weight-for-height greater than 3 standard deviations above the WHO Child Growth Standards median.

iii. Children aged between 5-19 years: Obesity is greater than 2 standard deviations above the WHO Growth Reference median.

New studies describe that the microenvironment in uterus defines the obesity of the future new born ${ }^{1}$ affecting in a higher proportion males. ${ }^{2}$ Animal models, ${ }^{3,4}$ have demonstrated the expression and suppression of genes associated to the mother's diet that affect the placental microenvironment ${ }^{5}$ and promotes obesity. These state correlates with chronic low-grade inflammation ${ }^{6,7}$ and immune system activation accompanied by insulin resistance. ${ }^{8}$ On the long run, there is a long (intra uterus-pre-puberty-puberty-post puberty), ${ }^{9}$ proinflammatory environment on germ cells.

\section{Childhood and puberty}

In childhood obesity is associated with a higher chance of adult obesity, premature death, and disability. ${ }^{10}$ Obese children is associated with endocrine abnormalities, like exaggerated adrenarche, hyperandrogenism as well as an increased risk of polycystic ovary syndrome, these events impact at hypothalamus-hypophysis-gonadal axis and fertility. ${ }^{11,12}$ There is also extensive evidence suggesting that excess adiposity during childhood influence at pubertal development. ${ }^{13}$ Excess body weight during childhood influence at pubertal development, on a timing effect of pubertal onset and hormone levels related to kisspeptin and metabolism. ${ }^{14}$

Puberty appearance in obese boys and girls has also been associated with abnormal levels of adrenal and gonadal hormones. Levels of dehydroepiandrosterone sulphate (DHEAS) have been directly associated with body weight in 8-year-old children, where they could contribute to the association between early growth and adult disease risk, by enhancing insulin resistance and central fat deposition. ${ }^{15}$ Obese peripubertal girls show significant hyperandrogenism, by increased levels of total testosterone and free testosterone and decreased sex steroid-binding globulin (SHBG) compared with nonobese patients. ${ }^{16}$ Insulin and LH contribute to increased testosterone in obese peripubertal adolescents, although other factors associated with obesity may also mediate this association. Obesity is associated with low overnight LH pulse frequency in prepubertal and early pubertal girls whereas by Tanner stages $3-5$, LH frequency is 
abnormally elevated in obese girls, possibly reflecting the effects of hyperandrogenism and resembling findings of adult polycystic ovary syndrome. Interestingly, weight loss has been associated with a significant decrease in testosterone concentrations in obese boys and girls. ${ }^{16}$ These suggest that childhood obesity contribute to the appearance of endocrine disturbances during adolescence, and increase the risk of developing infertility later in life.

\section{Adult male fertility affections associated to obesity}

The adult age male need to complete a functional spermatogenesis, these process is highly complex and specialized involved various mechanisms (hypothalamus, pituitary, Leydig cells, Sertoli cells and sex steroids. ${ }^{17}$ Hypothalamic-pituitary-gonadal (HPG) axis is vital for the reproductive function, and can be dysregulated with obesity. There is a direct correlation between hypogonadism and obesity, ${ }^{18}$ due to higher estrogen levels and hypoandrogenemia, affecting aromatase receptors and Leydig and Sertoli behavior. ${ }^{19}$ In obesity, testosterone is metabolized to estradiol by the cytochrome P450 enzyme aromatase in adipose tissue, which elevate estrogens levels. ${ }^{20}$ The increased of estrogens negative feedback upon the HPG axis via kisspeptin neurons, ${ }^{18}$ result in a down regulation of testosterone and thus spermatogenesis. ${ }^{21}$ Obesity promotes expression of proinflammatory cytokines like TNF and IL-6 that down regulate testosterone levels while endocrine changes modify concentration of insulin, sex-hormone-binding-globulin (SHBG), leptin, and inhibin $\mathrm{B}$, all affecting free testosterone levels.

Testosterone is involved in insulin regulation, metabolism of lipids and body composition. ${ }^{22}$ Hyperinsulinemia has been shown to have a negative effect on spermatogenesis with a significantly higher level of nuclear and mitochondrial DNA damage. At the same time, increased concentration of estrogen diminishes SHBG levels. ${ }^{22}$ Leptin is a hormone secreted by adipocytes to regulate satiety, but is also involved in sexual maturation and reproduction. ${ }^{20}$ Leptin stimulates GnRH release; in obesity, excess leptin cause a resistance later in life. ${ }^{18}$ The production of inhibin B by Sertoli cells is the most effective marker for normal spermatogenesis. Inhibin B is a growth-like factor which acts in the testes to inhibit FSH production and to stimulate testosterone production by Leydig cells. ${ }^{23}$ The reduced levels of inhibin B found in obese males is indicative of seminiferous tubule dysfunction and is hypothesized to be due to a lower number of Sertoli cells $;{ }^{20}$ however, a compensatory increase in FSH levels in response to low inhibin $\mathrm{B}$ has not been observed, indicating potential partial dysregulation of the HPG axis..$^{23}$ Obesity cause a chronic inflammatory state causing formation of reactive oxygen species (ROS) which can induce damage to sperm DNA and membrane as well as increase stress on testicular environment. ${ }^{20}$ Obesity during childhood increases the insult time creating more profound damage on DNA fragmentation; that affect fertilization and embryonic development. ${ }^{22}$

\section{Adult female fertility affections associated to obesity}

In adult age, female need to complete multiple reproductive functions like: liberate the oocyte, complete the meiosis, fertilization, implantation, and pregnancy development. All these processes are affect by obesity secondary a dysregulation at the Hypothalamicpituitary-gonadal (HPG) axis. It is associated with a higher level of proinflammatory cytokines in follicular fluid. ${ }^{24} \mathrm{~A}$ direct correlation is established between hypogonadism and obesity ${ }^{18}$ due to higher levels of estrogen and hypoandrogenemia that affect aromatase receptors and granulose cell behavior, causing anovulation and endometrial receptivity.

Ovulation disorders; Most of the ovulation disorders at obese patients are oligo or anovulation. The endocrine activity of the hypothalamus-hypophysis-ovary axis in the prepuberal female will remain dormant until she reaches a critical weight and composition, liberating pulsate of kisspeptin and secondly FSH-LH activation that marks the onset of puberty continue at their reproductive live. ${ }^{25}$ Obesity is associated with polycystic ovarian syndrome (PCOS) which is marked by hyperandrogenemia. Hyperandrogenemia induce apoptosis in granulose cell, dysregulate pituitary function due to increased aromatase activity in peripheral tissues and an increases negative feedback on gonadotrophin secretion. At the same time insulin resistance is associated with ovarian steroidogenesis and a decrease of sex hormone-binding globulin and leptin levels. ${ }^{26}$ Hormonal alterations associated to obesity affect endometrial with a higher incidence of endometrial cancer in obese patients. ${ }^{10}$ Animal models have shown that leptin and leptin receptor (LEPR) play a relevant role in the regulation of implantation. Obesity disrupts leptin/ LEPR which may disturb endometrial receptivity and implantation leading to impaired fecundity. The effects of leptin on reproduction are not homogenous, and both stimulatory and inhibitory functions have been described. ${ }^{27,28}$ Although it is known that leptin has a complex role in endometrium functionality basic science and clinical studies area necessary to comprehend the effect of obesity on implantation and early pregnancy.

\section{Conclusion}

Obesity is a worldwide problem that affect all ages, the impact at obesity and fertility involves multiples variants that creates a constant proinflammatory microenvironment in germ cell that have a negative impact on reproduction. We need to empathize the role of the hormone axis in both genders. In male increased BMI affect the testosterone levels and elevated ROS that damage sperm membrane and DNA. In women obesity modify the hormonal axis presenting ovulatory dysfunction and a decrease at endometrial receptivity affecting fertilization rate. Lifestyle modification is important to diminish inflammatory exposure and avoid long microenvironment deregulation on germ cells.

\section{Acknowledgements}

None.

\section{Conflict of interest}

Author declares there is no conflict of interest.

\section{References}

1. Oken E, Gillman MW. Fetal Origins of Obesit. Obes Res. 2003;11(4):496506.

2. Kozyrskyj AL, Kalu R, Koleva PT, et al. Fetal programming of overweight through the microbiome:boys are disproportionately affected. J Dev Orig Health Dis. 2016;7(1):25-34.

3. TD Jennings, MG Gonda, KR Underwood, et al. The influence of maternal nutrition on expression of genes responsible for adipogenesis and myogenesis in the bovine fetus. Animal. 2016;10(10):1697-1705 
4. Liping Qiao, Hyung sun Yoo, Alysha Madon, et al. Adiponectin Enhances Mouse Fetal Fat Deposition. Diabetes. 2012;61(12):3199-3207.

5. Lewis RM, Demmelmair H, Gaillard R, et al. The Placental Exposome: Placental Determinants of Fetal Adiposity and Postnatal Body Composition. Ann Nutr Metab. 2013;63(3):208-215.

6. Carolan E, Hogan AE, Corrigan M, et al. The Impact of Childhood Obesity on Inflammation, Innate Immune Cell Frequency, and Metabolic MicroRNA Expression. J Clin Endocrinol Metab. 2014;99(3):E474-478.

7. Jennifer Sacheck. Pediatric Obesity: An Inflammatory Condition? JPEN J Parenter Enteral Nutr.2008;32(6):633-637.

8. Esser N, Legrand-Poels S, Piette J, et al. Inflammation as a link between obesity, metabolic syndrome and type 2 diabetes. Diabetes Res Clin Pract. 2014;105(2):141-150.

9. Burt Solorzano CM, McCartney CR. McCartney Obesity and the pubertal transition in girls and boys. Reproduction. 2010;140(3): 399-410.

10. Jack A Yanovski. Pediatric obesity. An introduction. Appetite. 2015;93:312.

11. Davidson LM, Millar K, Jones C, et al. Deleterious effects of obesity upon the hormonal and molecular mechanisms controlling spermatogenesis and male fertility. Hum Fertil. 2015;18(3):184-193.

12. Pandey S, Pandey S, Maheshwari A, et al. The impact of female obesity on the outcome of fertility treatment. J Hum Reprod Sci. 2010;3(2):62-67.

13. Jeeson C Unni. Onset of Puberty in Relation to Obesity. Indian Pediatrics. 2016;53:379-380

14. Stephanie B. Seminara Fatness and fertility: which direction? J Clin Invest. 2014;124(7):2853-2854.

15. Dai YL, Fu JF, Liang L, et al. Association between obesity and sexua maturation in Chinese children: a muticenter study. Int $J$ Obes (Lond). 2014;38(10):1312-1316.

16. M-Loredana Marcovecchio, Francesco Chiarelli. Obesity and Growth during Childhood and Puberty. Shamir R, Turck D, Phillip M, editors. Nutrition and Growth. World Rev Nutr Diet: Basel; 2013. p 135-141.

17. Ruwanpura SM, McLachlan RI, Meachem SJ. Hormonal regulation of male germ cell development. Journal of Endocrinology, 2010;205(2):117131
18. Rao PM, Kelly DM, Jones TH. Testosterone and insulin resistance in the metabolic syndrome and T2DM in men. Nat Rev Endocrinol. 2013;9(8):479-483.

19. Reis LO, Dias FG. Male Fertility, Obesity, and Bariatric Surgery. Reproductive Sciences. 2012;19(8):778-785.

20. Du Plessis SS, Cabler S, McAlister DA, et al. The effect of obesity on sperm disorders and male infertility. Nature Reviews Urology, 2010;7(3):153-161.

21. MacDonald AA, Herbison GP, Showell M, et al. The impact of body mass index on semen parameters and reproductive hormones in human males: a systematic review with meta-analysis. Human Reproduction Update, 2010;16(3):293-311.

22. Chavarro JE, Toth TL, Wright DL, et al. Body mass index in relation to semen quality, sperm DNA integrity, and serum reproductive hormone levels among men attending an infertility clinic. Fertility and Sterility, 2010;93(7):2222-2231.

23. Palmer NO, Bakos HW, Fullston T, et al. Impact of obesity on male fertility, sperm function and molecular composition. Spermatogenesis. 2012;2(4);253-263

24. La Vignera S, Condorelli R, Bellanca S, et al. Obesity is associated with a higher level of pro-inflammatory cytokines in follicular fluid of women undergoing medically assisted procreation (PMA) programs. Eur. Rev. Med. Pharmacol. Sci. 2011;15(3);267-273.

25. Hussain MA, Song WJ, Wolfe A. There is Kisspeptin-and then there is Kisspeptin. Trends Endocrinol Metab. 2015;26(10):564-572.

26. Schisterman EF, Mumford SL, Browne RW, et al. Buck Louis Lipid Concentrations and Couple Fecundity: The LIFE Study J Clin Endocrinol Metab 2014;99(8):2786-2794.

27. Xian-Hua Lin, Miao-E. Liu, Hai-Yan Xu, et al. Leptin down-regulates g-ENaC expression: a novel mechanism involved in low endometrial receptivity. Fertil Steril. 2015;103(1):228-235.

28. Facts about overweight and obesity. 2017. 